\title{
Every DFS Tree of a 3-Connected Graph Contains a Contractible Edge
}

\author{
Amr Elmasry* $\quad$ Kurt Mehlhorn ${ }^{\dagger} \quad$ Jens M. Schmidt ${ }^{\ddagger}$
}

\begin{abstract}
Let $G$ be a 3-connected graph on more than 4 vertices. We show that every depth-first-search tree of $G$ contains a contractible edge. Moreover, we show that if $G$ is 3-regular or does not have two disjoint pairs of adjacent degree-3 vertices, every spanning tree of $G$ contains a contractible edge.
\end{abstract}

\section{Introduction}

A graph $G$ is connected if there exists a path between every two of its vertices. For $k>1$, $G$ is $k$-connected if deleting any $k-1$ of its vertices leaves a connected graph. In a $k$ connected graph every vertex has degree at least $k$. An edge in a 3-connected graph is contractible (also called 3-contractible) if its contraction results in a 3-connected graph.

Over 40 years ago, Tutte [Tut61] proved the fundamental result that every 3-connected graph on more than 4 vertices contains a contractible edge. Since then, the distribution of contractible edges in 3-connected graphs has been intensively studied. Many papers establish lower bounds on the number of contractible edges [AES87, Ota88, or on entire contractible subgraphs [Kri08]. See [Kri02 for an excellent survey. Bounds on the number of removable edges in 3-connected graphs [HJSW90, KWL07] have also been proved; an edge is removable if its removal leaves a 3 -connected graph.

In this paper, we strengthen Tutte's result by showing that every depth-first-search tree of a 3-connected graph contains a contractible edge. We also exhibit 3-connected graphs with a depth-first-search tree containing exactly one contractible edge, and 3-connected graphs with a spanning tree containing no contractible edge. We call a 3-connected graph a fox if it has a spanning tree containing no contractible edge. We present infinite families of foxes and give conditions under which a 3-connected graph is not a fox.

A certifying algorithm for 3-connectivity returns a proof (a certificate) for the 3connectivity of the input graph, which can be verified efficiently. See [KMMS06] and [MNU99, Section 2.14] for a general discussion of certifying algorithms. In [EMS10] we exploit the existence of a contractible edge in every depth-first-search tree to establish a linear-time

\footnotetext{
${ }^{*}$ MPI für Informatik, Supported by an Alexander von Humboldt Fellowship.

${ }^{\dagger}$ MPI für Informatik

${ }^{\ddagger}$ Dept. of Computer Science, FU Berlin. This research was supported by the Deutsche Forschungsgemeinschaft within the research training group "Methods for Discrete Structures" (GRK 1408).
} 
certifying algorithm for 3-connectivity of Hamiltonian graphs. The best certifying algorithm for 3-connectivity Sch10] of general graphs runs in time $O\left(n^{2}\right)$ and the best non-certifying decision algorithms [HT73, MR92] run in time $O(n+m)$, where $n$ is the number of vertices and $m$ is the number of edges of the underlying graph.

\section{Notations}

Let $G=(V, E)$ be an undirected graph with $n:=|V|$ and $m:=|E|$. For a vertex $x \in V$, let $\operatorname{deg}(x)$ be its degree in $G$. We denote an edge between vertices $x$ and $y$ by $x y$. If $x y \in E(G)$, we say that $x$ and $y$ are neighbors in $G$.

For any subset of vertices $V^{\prime} \subset V$, let $G \backslash V^{\prime}$ denote the graph resulting from $G$ by the removal of the vertices in $V^{\prime}$ and all their incident edges. A set of vertices whose removal disconnects the graph is called a vertex cut. If $V^{\prime}$ is a vertex cut of $G$, the connected components of $G \backslash V^{\prime}$ are called the split components (or separation classes) with respect to $V^{\prime}$. Vertex cuts of size one, two and three are called separation vertices, separation pairs and separation triples, respectively. Analogously, an edge cut of a graph $G$ is a set of edges whose removal disconnects $G$.

Let $x y$ be an edge of $G$. The contraction of $x y$ results in a graph $G^{\prime}=G / x y$ with vertex set $V\left(G^{\prime}\right)=V(G) \backslash\{x, y\} \cup\left\{v_{x y}\right\}$, where $v_{x y}$ is a new vertex. The edge $x y$ is removed and for all edges having exactly one endpoint in $\{x, y\}$, this vertex is replaced by $v_{x y}$. Finally, only one edge of each set of parallel edges is kept.

\section{Preliminaries}

We use the following known results in our proofs.

Fact 1.1 An edge $x y$ in a 3-connected graph with $n>4$ is contractible if and only if no separation triple containing $x$ and $y$ exists.

Theorem 1.2 (Tutte [Tut61]) Every 3-connected graph with $n>4$ contains a contractible edge.

Lemma 1.3 (Halin [Hal69]) In a 3-connected graph with $n>4$, every vertex of degree 3 has an incident contractible edge.

Lemma 1.4 (Ota [Ota88]) Let $v$ be a vertex of degree 3 in a 3 -connected graph $G$ with $n>4$ and let $x, y$, and $z$ be its neighbors. If $x y \in E(G)$, then $v z$ is contractible.

\section{Separation Triples and Split Components}

We establish some properties of separation triples and split components. We shall use them later to prove our main results.

Lemma 2.1 Let $S T=\{x, y, z\}$ be a separation triple in a 3-connected graph $G$. Let $D$ be one of the split components of $G \backslash S T$. Then, every vertex in $S T$ has a neighbor in $D$. 
Proof. Assume otherwise, say $z$ has no neighbor in $D$. Then, $D$ is a split component of $G \backslash\{x, y\}$, a contradiction to $G$ being 3 -connected.

Lemma 2.2 Let $S T=\{x, y, z\}$ be a separation triple in a 3-connected graph $G$, and let $D$ be one of the split components of $G \backslash S T$. If $S T^{\prime}=\left\{x^{\prime}, y^{\prime}, z^{\prime}\right\}$ is a separation triple in $G$ with $S T^{\prime} \neq S T$ and $S T^{\prime} \subset V(D) \cup S T$, then there is a split component of $G \backslash S T^{\prime}$ properly contained in $D$.

Proof. Let $D, D_{1}, \ldots, D_{j}$ be the split components of $G \backslash S T$. Consider the components $D_{i}$, where $1 \leq i \leq j$. Every $D_{i}$ is connected in $G \backslash(V(D) \cup S T)$, and hence connected in $G \backslash S T^{\prime}$. Moreover, according to Lemma 2.1, any vertex in $S T \backslash S T^{\prime}$ has a neighbor in $D_{i}$. It follows that $\left(S T \backslash S T^{\prime}\right) \cup V\left(D_{i}\right)$ is contained in a split component of $G \backslash S T^{\prime}$. Since $S T \backslash S T^{\prime}$ is non-empty, $\left(S T \backslash S T^{\prime}\right) \cup \bigcup_{1<i<j} V\left(D_{i}\right)$ is contained in a split component of $G \backslash S T^{\prime}$. Any other split component of $G \backslash S T^{\prime}$ (there must be at least one) is contained in $V(G) \backslash\left(\left(S T \backslash S T^{\prime}\right) \cup \bigcup_{1 \leq i \leq j} V\left(D_{i}\right) \cup S T^{\prime}\right)$, and hence properly contained in $D$.

Lemma 2.3 Let $G$ be a 3-connected graph, and let $\{x, y, z\}$ and $\{v, y, w\}$ be two separation triples in $G$ intersecting exactly in $y$. Then, $v$ and $w$ are contained in the same split component of $G \backslash\{x, y, z\}$ if and only if $x$ and $z$ are contained in the same split component of $G \backslash\{v, y, w\}$. Moreover, if $v$ and $w$ belong to distinct split components, then each of $G \backslash\{x, y, z\}$ and $G \backslash\{v, y, w\}$ has exactly two split components.

Proof. Assume that $v$ and $w$ are contained in the same split component of $G \backslash\{x, y, z\}$. Then, there is a split component $S$ of $G \backslash\{x, y, z\}$ that contains a neighbor of $x$ and a neighbor of $z$, but neither $v$ nor $w$. As $S \cup\{x, z\}$ is connected in $G \backslash\{v, y, w\}, x$ and $z$ belong to the same split component of $G \backslash\{v, y, w\}$. Conversely, if $x$ and $z$ belong to the same split component of $G \backslash\{v, y, w\}$, then $v$ and $w$ belong to the same split component of $G \backslash\{x, y, z\}$ for the same reason. This proves the first claim.

Assume that there are more than two split components of $G \backslash\{x, y, z\}$. Then, among these split components there is a component containing neither $v$ nor $w$. It follows that $x$ and $z$ belong to the same split component of $G \backslash\{v, y, w\}$, and in accordance $v$ and $w$ belong to the same split component of $G \backslash\{x, y, z\}$. The same arguments apply if there are more than two split components of $G \backslash\{v, y, w\}$.

We call two separation triples $\{x, y, z\}$ and $\{v, y, w\}$ crossing, if they intersect in exactly one vertex and if $v$ and $w$ belong to distinct components of $G \backslash\{x, y, z\}$. Then, $x$ and $z$ belong to distinct components of $G \backslash\{v, y, w\}$ by Lemma 2.3. In addition, $G \backslash\{x, y, z\}$ and $G \backslash\{v, y, w\}$ both have exactly two split components.

Lemma 2.4 Let $G$ be a 3-connected graph, let $\{x, y, z\}$ and $\{v, y, w\}$ be two crossing separation triples in $G$, let $D$ be the split component of $G \backslash\{x, y, z\}$ containing $v$, and let $X$ and $Z$ be the split components of $G \backslash\{v, y, w\}$ containing $x$ and $z$, respectively. Then, either $X \cap D=\emptyset$ or $\{x, y, v\}$ is a separation triple. Also, either $Z \cap D=\emptyset$ or $\{z, y, v\}$ is a separation triple. 


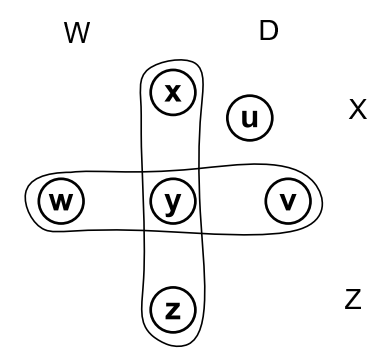

Figure 1: Two crossing separation triples.

Proof. Assume $X \cap D \neq \emptyset$. Consider any edge $u r \in E(G)$ with $u \in V(X \cap D)$ and $r \notin V(X \cap D)$. See Figure 1. Then, $r \in\{x, y, z, v, w\}$. However, $r \neq z$ because $\{v, y, w\}$ separates $X$ from $z$, and $r \neq w$ because $\{x, y, z\}$ separates $D$ from $w$. It follows that $\{x, y, v\}$ separates $X \cap D$ from the rest of $G$. Analogously, if $Z \cap D \neq \emptyset$, then $\{z, y, v\}$ separates $Z \cap D$ from the rest of $G$.

Lemma 2.5 Let $G$ be a 3-connected graph, let $S T=\{v, y, w\}$ be a separation triple in $G$, and let $X$ be a split component of $G \backslash S T$. If $G \backslash X$ is not 2-connected and $a$ is a separation vertex of $G \backslash X$, then $a \notin S T$ and one of the vertices in $S T$ has a as its only neighbor in $G \backslash X$ (and hence is a split component of $G \backslash(V(X) \cup\{a\})$ ). Conversely, if each vertex in $S T$ has at least two neighbors in $G \backslash X$, then $G \backslash X$ is 2-connected.

Proof. Assume that $G \backslash X$ is not 2-connected. Then, there is a separation vertex $a$ that splits $G \backslash X$. If one of the split components of $G \backslash(V(X) \cup\{a\})$ does not contain a vertex from $S T$, then $a$ is a separation vertex in $G$, contradicting $G$ being 3 -connected. It follows that every split component of $G \backslash(V(X) \cup\{a\})$ contains at least one vertex from $S T$. If $a \in S T$, say $a=y$, then $G \backslash(V(X) \cup\{a\})$ has exactly two split components one containing $v$ and one containing $w$. Since $S T$ is a separation triple in $G$, there are vertices in $G \backslash X$ other than those in $S T$. It follows that one of the components of $G \backslash(V(X) \cup\{a\})$ must have at least two vertices, say the component containing $w$. Then, $\{y, w\}$ splits $G$, a contradiction to $G$ being 3-connected. Therefore, $a \notin S T$.

The vertices of $S T$ cannot all lie in one split component of $G \backslash(V(X) \cup\{a\})$. Hence, at least one of these split components, say $S$, contains exactly one vertex from $S T$, say $w$. If $w$ has a neighbor in $G \backslash X$ other than $a$, then $|V(S)|>1$ and $S \backslash\{w\}$ is a split component of $G \backslash\{a, w\}$, contradicting $G$ being 3-connected.

\section{Contractible Edges and Spanning Trees}

We next give a sufficient condition for every spanning tree of a 3-connected graph to contain a contractible edge.

Lemma 3.1 Let $G$ be a 3-connected graph with $n>4$, and let $F$ be an edge cut of $G$. If every edge $e$ in $F$ has an end vertex $x$, where $\operatorname{deg}(x)=3$ and $x$ has two neighbors in $G \backslash F$ adjacent to each other, then $G$ is not a fox. 


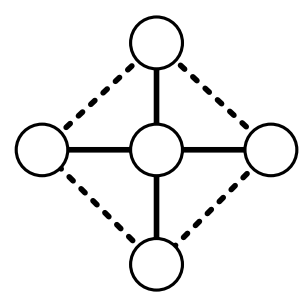

(a) $W_{5}$

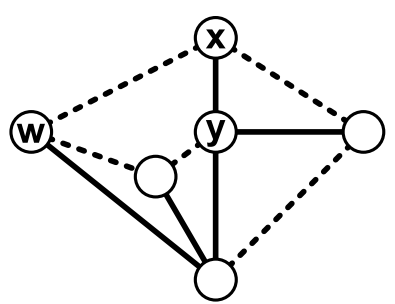

(b) $n=6$

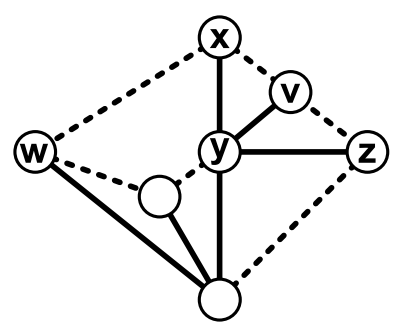

(c) $n=7$

Figure 2: The solid edges are non-contractible and form a spanning tree.

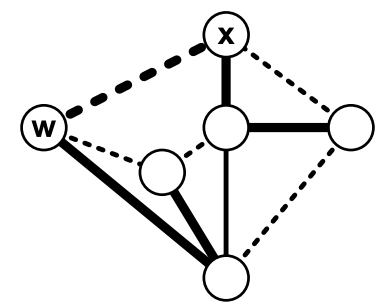

Figure 3: A depth-first-search tree (thick edges) with only one contractible edge, namely $x w$.

Proof. Let $v$ and $w$ be the two neighbors of $x$ in $G \backslash F$. Since $v$ and $w$ are adjacent, Lemma 1.4 implies that $e$ is contractible. Therefore, every edge in $F$ is contractible, and hence every spanning tree of $G$ contains at least one contractible edge.

Examples: There are arbitrary large foxes; the wheel graphs $W_{i}, i \geq 5$, with the spokes as the spanning tree form an infinite family, see Figure 2(a). Figure 2(b) shows the base graph of another infinite family of examples. In this graph, the vertices $x, y$, and $w$ play a special role. The next larger graph in this family is obtained as follows: Let $v$ be the neighbor of $x$ that is neither $y$ nor $w$ in the smaller graph, subdivide $x v$ by one vertex and connect the new vertex with $y$; see Figure 2(c).

We will show that every depth-first-search tree of a 3-connected graph contains a contractible edge. The graph on 6 vertices of Figure 3 shows that this bound is tight. However, we are not aware of any graph on more than 6 vertices that admits a depthfirst-search tree containing exactly one contractible edge.

Consider a 3-connected graph $G$ with $n>4$. Assume that $G$ is a fox and let $T$ be a spanning tree of $G$ containing no contractible edge. It follows that for every edge $x y \in E(T)$ there exists a vertex $z \in V(G)$ such that $\{x, y, z\}$ is a separation triple. We call $\{x, y, z\}$ a $T$-separation triple. Split components that result from the removal of a $T$-separation triple are called $T$-split components. A $T$-split component is minimal if there is no $T$-split component properly contained in it. 


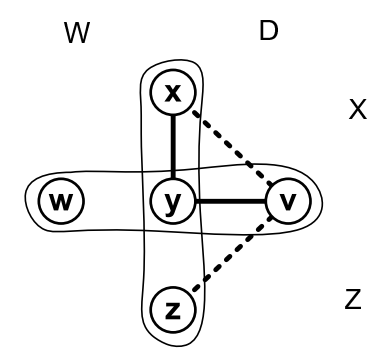

Figure 4: The $T$-minimal split component $D$.

Lemma 3.2 Let $G$ be a 3-connected graph with $n>4$. Assume that $G$ is a fox and let $T$ be a spanning tree of $G$ containing no contractible edge. Then, every T-minimal split component consists of exactly one vertex, say $v$. This vertex has degree 3 and is incident to exactly one edge of $T$. More precisely, if the neighbors of $v$ in $G$ are $x, y$, and $z$ with $x y \in E(T)$, then $v z \notin E(T)$, and either $v x \in E(T)$ or $v y \in E(T)$.

Proof. Let $D$ be a $T$-minimal split component, and let $\{x, y, z\}$ with $x y \in E(T)$ be the associated separation triple. Since $T$ is a spanning tree, there exists a vertex $v \in V(D)$ that is a neighbor of $x, y$, or $z$ in $T$. We show that $D$ has only one vertex, namely $v$.

If $v z \in E(T)$, then $v z$ is non-contractible, and hence a separation triple $\{v, z, w\}$ exists. Since $x y \in E(G)$, either $w \in\{x, y\}$ or both $x$ and $y$ are in the same split component of $G \backslash\{v, z, w\}$. Consequently, there exists a split component $S$ of $G \backslash\{v, z, w\}$ such that $x, y \notin V(S)$. By Lemma 2.1, $v$ has a neighbor, say $u$, in $S$. Since $u \notin\{x, y, z\}, u$ is in the same split component of $G \backslash\{x, y, z\}$ as $v$, i.e. $u \in V(D)$. It follows that every vertex in $S$ is also in $D$. Since $v \notin V(S), S$ is properly contained in $D$, a contradiction to $D$ being minimal. It follows that $v z \notin E(T)$. Accordingly, either $v x \in E(T)$ or $v y \in E(T)$.

Assume w.l.o.g. that $v y \in E(T)$. See Figure 4. Therefore, $v y$ is non-contractible and a separation triple $\{v, y, w\}$ exists. If there is a split component of $G \backslash\{v, y, w\}$ containing neither $x$ nor $z$, the arguments of the preceding paragraph indicate that the $T$-split component $D$ is not $T$-minimal. It follows that $\{v, y, w\}$ splits $G$ into exactly two components, one containing $x$ and one containing $z$. Call the former component $X$ and the latter $Z$. We show next that both $X \cap D$ and $Z \cap D$ must be empty.

If $X \cap D \neq \emptyset$, Lemma 2.4 implies that $\{x, y, v\}$ separates $X \cap D$ from the rest of $G$, a contradiction to $D$ being minimal. This implies that $X \cap D=\emptyset$. Analogously, $Z \cap D=\emptyset$.

We have thus shown that, assuming $v \in V(G)$ is in a $T$-minimal split component, there exists a separation triple $\{x, y, z\}$ with $x y \in E(T)$, such that $v x, v y, v z \in E(G)$, $\operatorname{deg}(v)=3, v z \notin E(T)$ and w.l.o.g. $v y \in E(T)$.

It is interesting to note that although foxes must have some degree- 3 vertices as indicated by the previous lemma, not all vertices of a fox can be of degree 3 .

Theorem 3.3 If $G$ is a 3-connected 3-regular graph with $n>4$, then $G$ is not a fox.

Proof. Assume that $G$ has a spanning tree $T$ containing no contractible edge. According to Lemma 3.2, there are vertices $v, x, y, z \in V(G)$, such that $v x, v y, v z \in E(G), x y, v y \in$ 
$E(T)$ but $v z \notin E(T)$. Because $G$ is 3-regular, $\operatorname{deg}(x)=\operatorname{deg}(y)=3$. As $T$ is a spanning tree of $G$, either the third edge incident to $x$, say $x r$, or the third edge incident to $y$, say $y s$, is a tree edge. Since $v y \in E(G), x r$ is contractible by Lemma 1.4. Since $x y, v y \in E(T)$, both edges are non-contractible by assumption. Accordingly, ys is contractible by Lemma 1.3 . This contradicts the assumption that $T$ contains no contractible edge.

Consider a 3-connected graph $G$ with $n>4$. Assume that $G$ is a fox and let $T$ be a spanning tree of $G$ containing no contractible edge. Let $v$ be a $T$-minimal split component in $G$, and let $v y$ be the only tree edge incident to $v$. We call a $T$-separation triple $\{v, y, w\}$ a special T-separation triple. Split components that result from the removal of a special $T$-separation triple are called special $T$-split components. A special $T$-split component is minimal if there is no special $T$-split component properly contained in it.

Lemma 3.4 Let $G$ be a 3-connected graph with $n>4$. Assume that $G$ is a fox and let $T$ be a spanning tree of $G$ containing no contractible edge. Then, every special T-minimal split component consists of exactly one vertex and has a neighbor that is also a special $T$ minimal split component. Let $v$ and $v^{\prime}$ be such a pair of special T-minimal split components with $v v^{\prime} \in E(G)$. Then, there exists a vertex $y$ such that $v y, v^{\prime} y \in E(T)$.

Proof. Let $X$ be a minimal special $T$-split component; it is split off by the special $T$ separation triple $S T=\{v, y, w\}$ with $v$ being a $T$-minimal split component and $v y \in E(T)$. By Lemma 2.2, no other special $T$-separation triple has its three vertices in $V(X) \cup S T$. Since $S T$ is a $T$-separation triple, there exists a $T$-minimal split component $v^{\prime} \in V(X)$; $v^{\prime}$ belongs to a special $T$-separation triple $S T^{\prime}=\left\{v^{\prime}, y^{\prime}, w^{\prime}\right\}$ with $v^{\prime} y^{\prime} \in E(T)$, where $y^{\prime} \in V(X) \cup S T$ and $w^{\prime} \notin V(X) \cup S T$ (otherwise, $X$ would not be minimal).

Assume first that $y^{\prime} \in V(X)$. Then, $w^{\prime}$ must split $G \backslash X$, and Lemma 2.5 implies that one of the vertices in $S T$ has $w^{\prime}$ as its only neighbor in $G \backslash X$. Since $v y \in E(G)$, such vertex must be $w$. We next show that all neighbors of $w$ are contained in $S T^{\prime}$, and hence $w$ has degree 3 . Assume to the contrary that $w$ has a neighbor $u^{\prime} \notin S T^{\prime}$. Then, $u^{\prime}$ and $w$ belong to the same split component of $G \backslash S T^{\prime}$. Every path from $u^{\prime}$ to any vertex in a different split component of $G \backslash S T^{\prime}$ must pass through either $v^{\prime}, y^{\prime}$ or $w$. Hence, $\left\{v^{\prime}, y^{\prime}, w\right\}$ is a special $T$-separation triple contained in $V(X) \cup S T$. But such possibility is ruled out in the previous paragraph because of the minimality of $X$. It follows that $w$ has degree 3 , its neighbors are precisely the vertices in $S T^{\prime}$, and $w$ is a $T$-minimal split component. By Lemma 1.4. $w w^{\prime}$ is contractible, and accordingly does not belong to $T$. Also, $w v^{\prime} \notin E(T)$ since $v^{\prime} y^{\prime} \in E(T)$ and $v^{\prime}$ has only one incident tree edge. Hence, $w y^{\prime} \in E(T)$. Let $z^{\prime}$ be the third neighbor of $v^{\prime}$ besides $y^{\prime}$ and $w$. Then, $\left\{w, y^{\prime}, z^{\prime}\right\}$ is a special $T$-separation triple that separates $v^{\prime}$ from the rest of $G$, see Figure 5. This again contradicts our choice of $X$ being minimal. We conclude that $y^{\prime} \notin V(X)$, and hence $y^{\prime} \in S T$.

Since $v^{\prime}$ and $w^{\prime}$ are in different split components of $G \backslash S T$, using Lemma 2.3, the triples $S T$ and $S T^{\prime}$ cross. Hence, the vertices of $S T \backslash\left\{y^{\prime}\right\}$ must belong to different split components of $G \backslash S T^{\prime}$. Since $v y \in E(G)$, this excludes the possibility that $y^{\prime}=w$. Also, $y^{\prime} \neq v$, since otherwise $v$ would be incident to two tree edges, namely $v y$ and $v^{\prime} y^{\prime}$. It must then be the case that $y=y^{\prime}$. If $|V(X)|>1$, Lemma 2.4 implies that either $\left\{v^{\prime}, y, v\right\}$ or $\left\{v^{\prime}, y, w\right\}$ is a special $T$-separation triple. Such a triple has a split component properly 


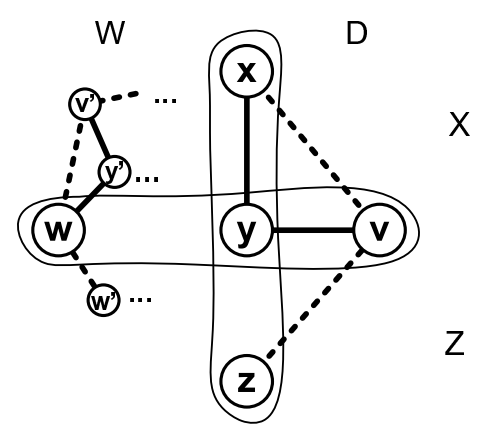

Figure 5: A case contradicting the minimality of $X$

contained in $X$, a contradiction to the minimality of $X$. It follows that $v^{\prime}$ is the only vertex in $X$. Let $w^{\prime \prime}$ be the third neighbor of $v$ besides $v^{\prime}$ and $y$. Then, $\left\{v^{\prime}, y, w^{\prime \prime}\right\}$ is a special $T$-separation triple that separates $v$ from the rest of $G$. We conclude that $v$ and $v^{\prime}$ are both special $T$-minimal split components, $v v^{\prime} \in E(G)$ and $v y, v^{\prime} y \in E(T)$.

Theorem 3.5 Let $G$ be a 3-connected graph with $n>4$. Assume that $G$ is a fox and let $T$ be a spanning tree of $G$ containing no contractible edge. Then, there exist two edges in $G$ such that their four end vertices are distinct special $T$-minimal split components.

Proof. Let $v$ and $v^{\prime}$ be adjacent special $T$-minimal split components as in Lemma 3.4 $v$ is split off by $S T^{\prime}=\left\{v^{\prime}, y, w^{\prime}\right\}$ and $v^{\prime}$ is split off by $S T=\{v, y, w\}$.

Assume first that there is a special $T$-minimal split component in $V(G) \backslash\left\{v, v^{\prime}, y, w, w^{\prime}\right\}$. Call it $z$, and let $z^{\prime}$ be the adjacent special $T$-minimal split component. Then, $z^{\prime} \notin\left\{v, v^{\prime}\right\}$, and hence $\left(v, v^{\prime}\right)$ and $\left(z, z^{\prime}\right)$ are the desired pairs.

Otherwise, any special $T$-minimal split component of $G$ is contained in $\left\{v, v^{\prime}, y, w, w^{\prime}\right\}$. Let $W^{\prime}$ be the component of $G \backslash S T$ containing $w^{\prime}$, and let $W$ be the component of $G \backslash S T^{\prime}$ containing $w$. Both $W^{\prime}$ and $W$ are special $T$-split components, and hence contain special $T$-minimal split components. These components must be $w$ for $W$ and $w^{\prime}$ for $W^{\prime}$. Then, $\left(v, w^{\prime}\right)$ and $\left(v^{\prime}, w\right)$ are the desired pairs.

Next, we use Theorem 3.5 to prove our main result.

Theorem 3.6 Consider a 3-connected graph $G$ with $n>4$. Every depth-first-search tree of $G$ contains a contractible edge.

Proof. Let $T$ be a depth-first-search tree of $G$, and assume that $T$ contains no contractible edge. By Theorem 3.5, there exist two pairs of distinct degree-3 vertices, each vertex is a $T$-minimal split component, such that the vertices of each pair are adjacent in $G$. By Lemma 3.2 , every $T$-minimal split component is a degree-3 vertex that is either the root or a leaf in $T$. Accordingly, there exists a pair of vertices that are leaves in $T$ while being adjacent in $G$, a contradiction to the fact that $T$ is a depth-first-search tree. 


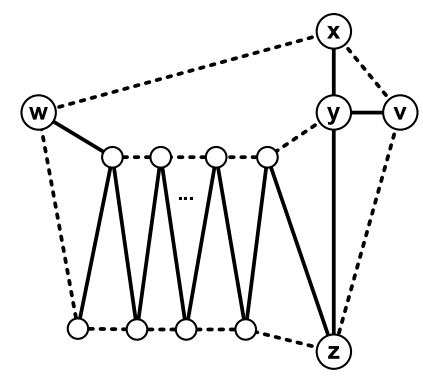

Figure 6: A fox with exactly four degree-3 vertices.

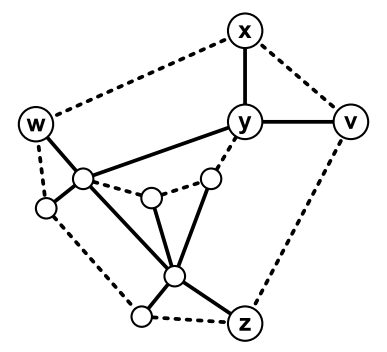

Figure 7: A fox with $m=2 n-3$.

We remark that there are arbitrarily large foxes having exactly four vertices of degree 3, see Figure 6.

\section{Conclusions}

Our main result is that every depth-first-search tree of a 3-connected graph contains a contractible edge. However, not every spanning tree of a 3-connected graph contains a contractible edge. We hope that our positive result will lead to a linear-time certifying algorithm for three-connectivity.

Foxes, i.e. 3-connected graphs with a spanning tree that contains no contractible edge, are interesting from a combinatorial view. We wonder if there is an inductive characterization of foxes. All Wheel graphs, as well as the foxes in Figure 2, satisfy the equation $m=2 n-2$. Figure 7 depicts a fox with $m=2 n-3$.

\section{References}

[AES87] K. Ando, H. Enomoto, and A. Saito. Contractible edges in 3-connected graphs. J. Comb. Theory Ser. B, 42(1):87-93, 1987.

[EMS10] A. Elmasry, K. Mehlhorn, and J. M. Schmidt. Every DFS tree of a 3-connected graph contains a contractible edge. (Available at the authors home pages), February 2010. 
[Hal69] R. Halin. Zur Theorie der n-fach zusammenhängenden Graphen. Abhandlungen aus dem Mathematischen Seminar der Universität Hamburg, 33(3):133164, 1969.

[HJSW90] D. A. Holton, B. Jackson, A. Saito, and N. C. Wormald. Removable edges in 3-connected graphs. J. Graph Theory, 14(4):465-473, 1990.

[HT73] J. E. Hopcroft and R. E. Tarjan. Dividing a graph into triconnected components. SIAM J. Comput., 2(3):135-158, 1973.

[KMMS06] D. Kratsch, R. M. McConnell, K. Mehlhorn, and J. P. Spinrad. Certifying algorithms for recognizing interval graphs and permutation graphs. SIAM J. Comput., 36(2):326-353, 2006. preliminary version in SODA 2003, pp. 158167.

[Kri02] M. Kriesell. A survey on contractible edges in graphs of a prescribed vertex connectivity. Graphs and Combinatorics, 18(1):1-30, 2002.

[Kri08] M. Kriesell. On the number of contractible triples in 3-connected graphs. J. Comb. Theory Ser. B, 98(1):136-145, 2008.

[KWL07] H. Kang, J. Wu, and G. Li. Removable edges of a spanning tree in 3-connected 3-regular graphs. In Frontiers in Algorithmics, LNCS 4613, pages 337-345, 2007.

[MNU99] K. Mehlhorn, S. Näher, and C. Uhrig. The LEDA Platform of Combinatorial and Geometric Computing. Cambridge University Press, 1999.

[MR92] G. L. Miller and V. Ramachandran. A new graph triconnectivity algorithm and its parallelization. Combinatorica, 12(1):53-76, 1992.

[Ota88] K. Ota. The number of contractible edges in 3-connected graphs. Graphs and Combinatorics, 4(1):333-354, 1988.

[Sch10] J. M. Schmidt. Construction sequences and certifying 3-connectedness. In 27th International Symposium on Theoretical Aspects of Computer Science (STACS'10), Nancy, France, 2010. (to appear).

[Tut61] W. T. Tutte. A theory of 3-connected graphs. Indag. Math., 23:441-455, 1961. 